OPEN

SUBJECT AREAS:

METABOLISM

EVOLUTIONARY

DEVELOPMENTAL

BIOLOGY

EVOLUTIONARY ECOLOGY

Received

27 September 2013

Accepted

13 November 2013

Published

29 November 2013

Correspondence and requests for materials should be addressed to F.A. (faubret@gmail.

com)

\section{Heart rates increase after hatching in two species of natricine snakes}

\author{
Fabien Aubret \\ Station d'Ecologie Expérimentale de Moulis, CNRS, 09200 Moulis, France.
}

Experimental studies have shown heart rates to decrease from embryo to hatchling stage in turtles, remain steady in skinks, and increase in birds. However, no snake species has been studied in this regard. I recorded heart rate evolution trajectories from embryo to juvenile stage in 78 eggs from two species of European Natricine snakes. Unexpectedly, snakes behaved more like birds than turtles or lizards: heart rates increased after hatching in both $N$. maura and $N$. natrix, respectively by $43.92 \pm 22.84 \%$ and $35.92 \pm 24.52 \%$. Heart rate shift was not related to an abrupt elevation of metabolism per se (snakes that increased their heart rates the most sharply grew the least after birth), but rather due to a number of smaller eggs that experienced lower than normal heart rates throughout the incubation and recovered a normal heart rate post-birth. This finding is discussed in the light of hatching synchrony benefits.

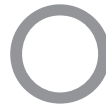

ntogenetic shifts in heart rates from embryo to hatchling stage are known to occur in a variety of amniotic vertebrates, in both ectotherms and endotherms. Such shifts may translate into a drop in heart rate (in turtles ${ }^{1-4}$ ), little or no change (in skinks ${ }^{5,6}$ ) or an increase in heart rate (in birds ${ }^{7}$ ). These shifts in heart rates may reflect the tremendous physiological changes accompanying hatching, from respiration, locomotion, through to energetic independence of the newly hatched organism ${ }^{8}$. However, why the direction and intensity of changes varies amongst groups remains an open question.

In birds, Pearson \& Tazawa ${ }^{7}$ suggested that the ontogenetic shift in heart rate may be explained by the development of endothermic metabolism, hence an elevation in metabolic rate. In the emydid turtles Chrysemys picta and Graptemys pseudogeographica kohniiturtles, it was proposed that a drop in heart rate from embryo to hatchling may be adaptive $e^{4}$. That is, while developing embryos may benefit from accelerated developmental rate (earlier hatching typically enhances offspring fitness ${ }^{9,10}$ ), young turtles no longer have a guaranteed food supply (i.e. yolk contained within the egg). Young turtles thus benefit from a lower post-hatching metabolic rate presumably in order to avoid fast depletion of resources in the face of uncertain levels of food availability ${ }^{4}$. Squamate reptiles such as the three-lined skink (Bassiana duperreyi) and the eastern fence lizards (Sceloporus undulatus) seem to show a different pattern, with little or no ontogenetic shift in heart rates from embryo to hatchling stages ${ }^{5,6}$. Du et al. ${ }^{4}$ explained this lack of consistency by the fact that annual survival rates average much higher in turtles than in squamates ${ }^{11}$. As a result, short-lived lizards (i.e. compared to turtles) may adopt "riskier" life-history tactics, expending energy at high rates despite the uncertainty about future food availability ${ }^{12}$.

Clearly, the current scarcity of experimental data, both within and amongst amniotic taxa, greatly limits our understanding of the physiological processes involved in over-birth shifts in heart rates, their interactions with life history traits (i.e. sex, body size, growth rates), and ultimately the adaptive value of such shifts. Further, to my knowledge, no snake species has previously been studied in this regard, so that no general pattern in heart rates can be yet defined amongst squamates.

The purpose of this study was thus to advance our knowledge in this regard, with special emphasis given to the physiological aspects of heart rate variations from embryo to hatchling. I recorded heart rate evolution trajectories from embryo to juvenile stage in 78 eggs from two species of European natricine snakes (the viperine snake Natrix maura and grass snake Natrix natrix) so as to precisely document the potential interactions amongst species, sex, egg mass, hatchling body size, post-hatching growth rates and resting metabolism.

\section{Results}

Trait comparison between sexes, species and interactions. Egg mass. A two factor repeated measures ANOVA with species and sex as factors and the five successive egg masses as repeated measures yielded $\mathrm{F}_{4,284}=1.80 ; \mathrm{P}=$ 0.13 (global interaction term; see Table 1). There was no effect of sex on egg mass evolution trajectories. N. natrix eggs were heavier at oviposition, and grew at higher rates than $N$. maura eggs (14.95 $\pm 6.76 \%$ increase in mass 
Table 1 Statistical results of a repeated measure ANOVA with species and sex as factors and egg mass as repeated variable

\begin{tabular}{lrcccl} 
Effect & SS & Df & MS & $F$ & $P$ \\
\hline Species & 2.368 & 1 & 2.368 & 51.94 & $\mathbf{0 . 0 0 0 1}$ \\
Sex & .052 & 1 & 0.052 & 1.13 & 0.291 \\
Species * Sex & .023 & 1 & 0.023 & 0.50 & 0.482 \\
Egg mass & .058 & 4 & 0.014 & 34.89 & $\mathbf{0 . 0 0 0 1}$ \\
Egg mass * Species & .035 & 4 & 0.009 & 21.19 & $\mathbf{0 . 0 0 0 1}$ \\
Egg mass * Sex & .001 & 4 & 0.001 & 0.49 & 0.739 \\
Egg mass * Species * Sex & .003 & 4 & 0.001 & 1.80 & 0.129 \\
\hline
\end{tabular}

versus $4.10 \pm 6.65 \%$ respectively; $\mathrm{P}<0.001$ ) throughout the incubation period. Maximum egg mass was reached around day 28 of the incubation period in both species (Fig. 1). I calculated a mean egg mass, averaged across the incubation period, for each individual within each species.

Heart rates. A two factor repeated measures ANOVA with species and sex as factors and the five successive heart rate readings as repeated measures yielded $\mathrm{F}_{4,284}=0.47, \mathrm{P}=0.76$ (global interaction term; see Table 2). There was no difference in heart rates between sexes. Heart rates changed throughout the incubation period and after hatching $(\mathrm{P}<0.001)$. Heart rate trajectories were comparable between species (see Fig. 2). There was, however, an interaction between heart rate readings across time and species $(\mathrm{P}<0.001$; see Fig. 2), possibly due to an initial difference in heart rates between $N$. natrix and $N$. maura (incubation time $=10$ days). Post-hoc analysis (Tukey HSD) confirmed that heart rates only differed between species at egg age $=10$ days $(\mathrm{P}<0.001)$. I calculated mean embryo heart rates, averaged across the incubation period, for each individual within each species.

Heart rates, egg size and body size. $N$. maura egg mass at oviposition largely explained hatchling body size (body mass, snout-vent length and BCI (Body Condition Index; see methods for details); Linear Regressions; all $\mathrm{R}>0.47$; all $\mathrm{P}<0.006$ ), while $N$. natrix egg mass at oviposition positively correlated with hatchling body mass and snout-vent length (both $\mathrm{R}>0.88$; both $\mathrm{P}<0.001$ ), but

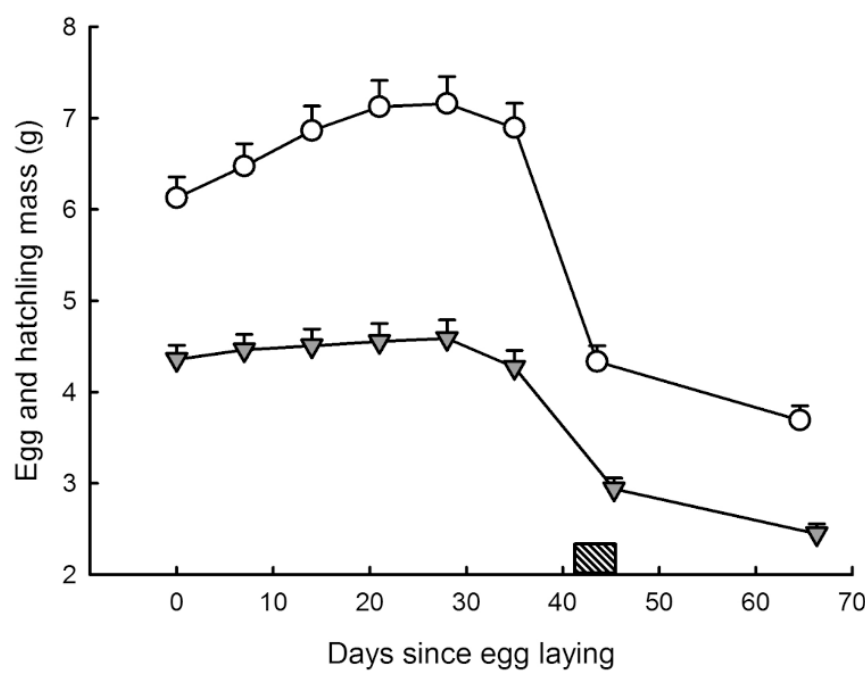

Figure $1 \mid$ Mass evolution was measured in 28 eggs and hatchlings of the grass snake $N$. natrix (open circles) and 51 eggs and hatchlings Viperine snake $N$. maura (grey triangles). A repeated measure ANOVA with species as factor and egg/hatchling mass as the repeated measure over time yielded $\mathrm{F}_{7,469}=7.38 ; \mathrm{P}<0.001$. Egg masses were measured every week. Snake masses were measured at birth and 3 weeks old. Snakes were unfed. Means \pm SE are plotted.
Table 2 | Statistical results of a repeated measure ANOVA with species and sex as factors and heart rates as repeated variable

\begin{tabular}{lccccl} 
Effect & SS & Df & MS & $F$ & $P$ \\
\hline Species & 0.006 & 1 & 0.006 & 1.27 & 0.263 \\
Sex & 0.001 & 1 & 0.001 & 0.27 & 0.603 \\
Species * Sex & 0.008 & 1 & 0.008 & 1.84 & 0.179 \\
Heart rate & 1.143 & 4 & 0.286 & 93.36 & $\mathbf{0 . 0 0 0 1}$ \\
Heart rate * Sex & 0.001 & 4 & 0.001 & 0.04 & 0.997 \\
Heart rate * Species & 0.089 & 4 & 0.022 & 7.23 & $\mathbf{0 . 0 0 0 1}$ \\
Heart rate * Sex* Species & 0.006 & 4 & 0.001 & 0.47 & 0.761 \\
\hline
\end{tabular}

not with $\mathrm{BCI}(\mathrm{R}=0.36 ; \mathrm{P}=0.057)$. Embryo mean heart rates were not correlated with incubation duration in either species (both $\mathrm{R}<$ 0.17; both $\mathrm{P}>0.25$ ). Embryo mean heart rates were, however, positively correlated to mean egg mass in $N$. natrix $(\mathrm{R}=0.54 ; \mathrm{P}=$ 0.003; Fig. 3) but not in N. maura ( $\mathrm{R}=0.01 ; \mathrm{P}=0.99)$. Post-hatching heart rates were unrelated to hatchling body size in $N$. natrix (all $\mathrm{R}<$ 0.24 ; all $\mathrm{P}>0.21)$. In $N$. maura however, post-hatching heart rates were positively correlated with $\operatorname{SVL}(\mathrm{R}=0.37 ; \mathrm{P}<0.009)$, negatively correlated with $\mathrm{BCI}(\mathrm{R}=0.31 \mathrm{P}<0.034)$, but unrelated to body mass $(\mathrm{R}=0.01 \mathrm{P}=0.93)$.

Shift in heart rates and growth rates. In N. maura, the over-birth shift in heart rates was not related to concurrent growth rates in body mass $(\mathrm{R}=0.21 ; \mathrm{P}=0.15)$, but negatively correlated with growth rate in snout-vent length $(\mathrm{R}=0.34 ; \mathrm{P}<0.017)$. In $N$. natrix however, the shift in heart rates was not correlated with any of the body size traits or growth rates recorded (all $\mathrm{R}<0.31$; all $\mathrm{P}>0.11$ ). Surprisingly, there was a negative correlation between the relative amplitude of the shift in heart rates (\% of increase) and the mean heart rates across the incubation period (Fig. 4), both in $N$. maura $(\mathrm{R}=0.58 ; \mathrm{P}<0.001)$ and $N$. natrix $(\mathrm{R}=0.57 ; \mathrm{P}<0.002)$.

\section{Discussion}

This experimental study demonstrated that an ontogenetic shift in heart rates from embryo to hatchling stage may occur in Natricine

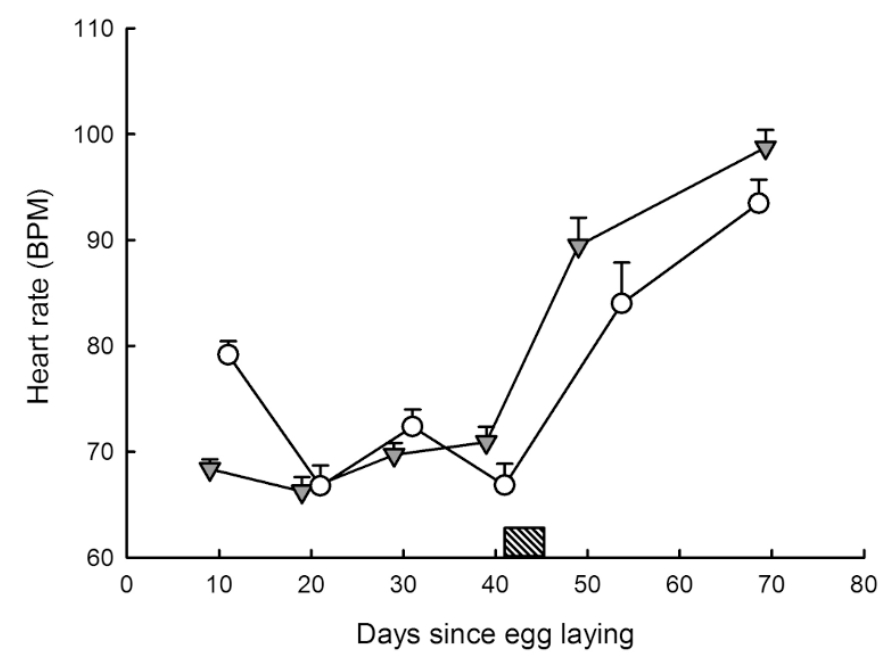

Figure $2 \mid$ Heart rates were measured in 28 eggs and hatchlings $N$. natrix (open circles) and 51 eggs and hatchlings $N$. maura (grey triangles). A repeated measure ANOVA with species as factor and heart beats as the repeated measure over time (every 10 days from laying and in hatchlings aged $24.35 \pm 3.74$ days) yielded: effect of species $\mathrm{F}_{1,76}=0.80, \mathrm{P}=0.38$; effect of time $\mathrm{F}_{4,304}=101.66, \mathrm{P}<0.001$; interaction term $\mathrm{F}_{4,304}=8.40, \mathrm{P}$ $<0.001$. Hatching occurred between the $41^{\text {st }}$ and $45^{\text {th }}$ days post laying (dash box). All measurements were made at a constant $28^{\circ} \mathrm{C}$. Means $\pm \mathrm{SE}$ are plotted. 


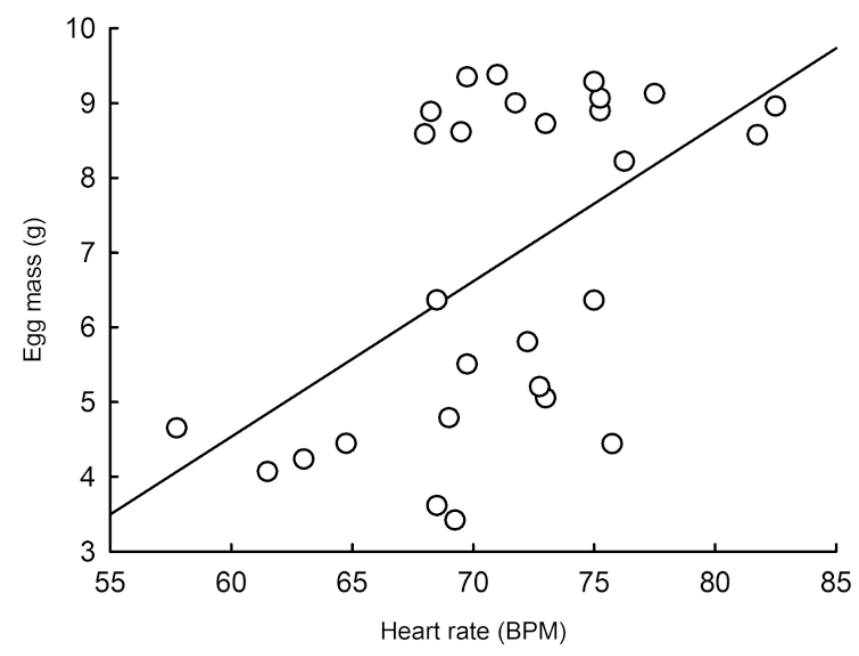

Figure $3 \mid$ Linear regression of mean embryo heart rates against mean egg mass in $28 N$. natrix eggs $\left(R=0.54 ; F_{1,26}=10.66 ; \mathrm{P}<0.003\right)$.

snakes, and, as in emydid turtles, rather late in embryogenesis (i.e. in the final quarter of the incubation period ${ }^{4}$ ). Further, the direction of the shift was opposite to that of turtles: heart rates sharply increased immediately after hatching with similar amplitude in both $N$. maura and N. natrix, respectively by $43.92 \pm 22.84 \%$ and $35.92 \pm 24.52 \%$. As such, these results are not only contrary to expectations based on previous studies of squamates reptiles (i.e. little or no shift in heart rates from embryo to hatchling ${ }^{5,6}$ ) but suggest that snake heart rates behaved more like birds (i.e. increase in heart rates) rather than turtles or lizards.

Given this unexpected result, one cannot exclude that some of the discrepancies found across relevant studies ${ }^{4-6}$ may stem from inconstant recording methods of metabolic rates in both embryos and hatchlings. For instance, while metabolic rates were in all cases recorded at similar temperatures in this study (between 28 and $30^{\circ} \mathrm{C}$ ), Angilletta et al. $^{5}$ estimated metabolic rates in the lizard Sceloporus undulatus using a flow-through system rather than via heart rate readings. Moreover, Radder \& Shine ${ }^{6}$ used, as in the current study, the Buddy ${ }^{\circledR}$ digital egg monitor, but anaesthetized weekold lizards, Bassiana duperreyi, with gaseous (Fluothane) inhalation prior to recording heart rates. On the other hand, the way Du et al. ${ }^{4}$ collected their data on emydid turtles was fully consistent with the

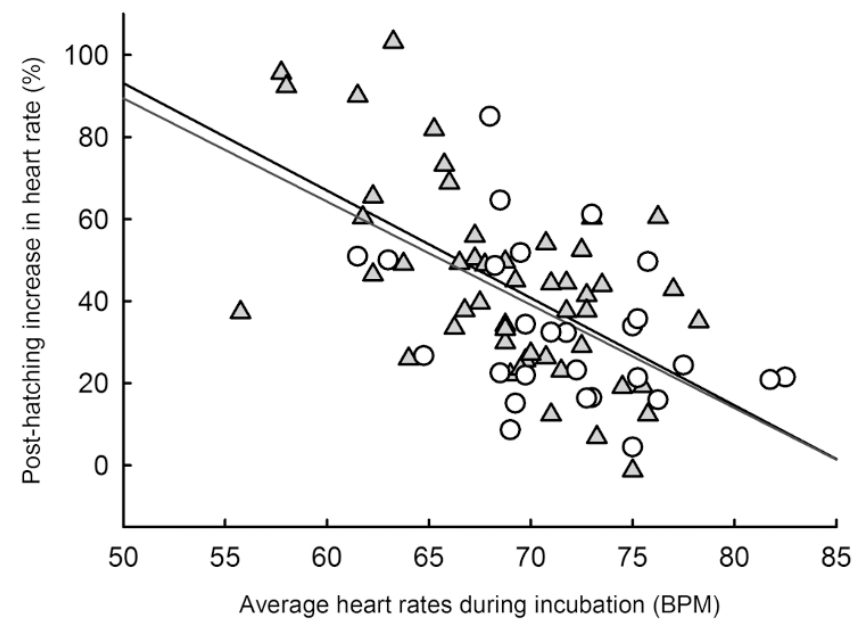

Figure $4 \mid$ Relative amplitude of the shift in heart rates (\% of increase) against heart rates averaged across the incubation period in $N$. natrix (open circles; $\mathrm{R}=0.57 ; \mathrm{F}_{1,26}=12.42 ; \mathrm{P}<0.002$ ) and $\mathrm{N}$. maura (grey triangles; $\left.\mathrm{R}=0.58 ; \mathrm{F}_{1,48}=24.75 ; \mathrm{P}<0.001\right)$. method applied to the current study. There is also a possibility that Natricine snakes are simply unusual in this regard and an exception amongst squamates. Further studies using consistent methods on a wider range of species will easily address these two possibilities. Nevertheless, this study provides new and challenging data to our current understanding, within and across amniotic groups, of the ontogenetic shift in heart rates from embryo to hatchling.

The bird ontogenetic shift in heart rates is explained by the development of endothermic metabolism, and hence an elevation in metabolic rate ${ }^{7}$. Thus, a sharp increase in heart rates may also presumably be related to a sudden shift in metabolic rates in young snakes. Yet, this was not supported by the data: the increase in heart rates was not related to growth rates in body mass recorded during the first 3 weeks after birth in both $N$. natrix and N. maura unfed snakes. In fact, the increase in heart rates was surprisingly negatively related to growth rates in snout-vent length: snakes that increased their heart rates the most sharply grew the least after birth. One striking result may provide some clues towards the explanation of this counter-intuitive finding: in both species and with similar amplitude, the shift in heart rates from embryo to hatchling was negatively correlated with heart rates throughout the incubation period. In other words, embryos that displayed slow heart rates during the incubation period strongly increased heart rates after birth (perhaps in a compensatory manner), while embryos that displayed already relatively high heart rates hardly shifted at all after birth (see Fig. 4). Further, as in turtles ${ }^{4}$, post-hatching heart rates in Natrix appear to be maintained through into adult life. Mean post-hatching heart rates were $98.74 \pm 12.15 \mathrm{BPM}$ in $N$. maura and $93.46 \pm$ 11.02 BPM in N. natrix. Such values are directly comparable with heart rates measured in sub-adult $N$. maura that maintained heart rates between 90 and $100 \mathrm{BPM}$ at $28^{\circ} \mathrm{C}$ body temperature ${ }^{13}$. This strongly suggests that high post-hatching heart rates in Natricine snakes are in fact "the norm". Some eggs seemingly experienced lower than normal heart rate values while incubating, and at birth, recovered normal heart rate values.

Are there any clues as to why some eggs may have incubated under sub-optimal conditions? Because the most drastic change between embryo and hatchling is a shift in respiration mode (i.e. through the egg shell versus lungs), one possibility is that some eggs experienced sub-optimal conditions during embryogenesis, perhaps due to impaired gas exchange due to egg positioning (for instance: embryo facing the substrate rather than the open air). Alternatively, it is striking that despite large differences in mean embryo heart rates amongst individual eggs (up to $40 \%$ in $N$. maura and $43 \%$ in $N$. natrix), incubation duration was unrelated to mean embryo heart rates but more so to egg mass at oviposition (i.e. because small eggs tended to have slower heart rates). This result is contrary to that found in other non-avian reptiles ${ }^{14}$ as well as in birds ${ }^{15}$ where embryonic heart rate (1) predicts incubation period and (2) is higher in species with smaller egg sizes and shorter incubation periods ${ }^{16}$.

In the current experiment, the data suggest that small eggs developed at slower rates than large eggs, presumably resulting in hatching synchrony: despite massive differences in egg size (up to $44 \%$ in N. maura and $195 \%$ in N. natrix), all eggs hatched within $45.38 \pm 1.55$ days for $N$. maura and $43.57 \pm 0.99$ days for $N$. natrix. Synchronized hatching is wide-spread amongst organisms ${ }^{17}$, including squamates ${ }^{18}$. Synchronized hatching may enhance offspring survival by diluting an individual's risk of predation or by simply swamping predators upon emergence ${ }^{19,20}$. Further studies are necessary to test whether or not, and via which mechanism, egg metabolism may be adjusted to relative egg size in order to promote hatching synchrony in snakes.

\section{Methods}

Study animals and study site. The viperine water snake (N. maura) occurs in France, Spain, Portugal, south-west Switzerland, north-west Italy and on a few Mediterranean islands ${ }^{21}$. This species is largely aquatic and individuals are always found in the 
vicinity of water ${ }^{22}$. Its close relative, the grass snake N. Natrix, is one of the most widely distributed snakes in Europe, ranging from southern England and Scandinavia to northern Africa and from the Atlantic Ocean to western Russia ${ }^{22}$. Study animals were caught along the banks of the Lez River and surrounding pasture and woodland in South-west Ariège, France. The study site was a twenty kilometre stretch of river including tributaries, between the localities of Moulis $\left(42^{\circ} 57^{\prime} 43^{\prime \prime} \mathrm{N} ; 1^{\circ} 05^{\prime} 30^{\prime \prime} \mathrm{E}\right)$ and Le pont $\left(42^{\circ} 52^{\prime} 32^{\prime \prime} \mathrm{N} 0^{\circ} 57^{\prime} 19^{\prime \prime} \mathrm{E}\right)$.

Animals used. A total of 16 gravid female viperine water snakes (hereafter N. maura) and 7 grass snakes (hereafter N. natrix) were captured in May and June 2011 and brought back to the laboratory (Station d'Ecologie Expérimentale à Moulis). Gravid females were housed individually in plastic containers $(60 \mathrm{~cm} \times 40 \mathrm{~cm} \times 15 \mathrm{~cm})$. A $40 \mathrm{~W}$ lamp fitted above each cage and set on a $12 / 12 \mathrm{~h}$ day and night timer provided heating opportunities. Each enclosure contained a $3 \mathrm{~cm}$ layer of moist peat, a clean water bowl, a shelter (terracotta roof tile) and an egg laying box. The egg laying box was a black plastic container $(15 \mathrm{~cm} \times 10 \mathrm{~cm} \times 5 \mathrm{~cm})$, with a $4 \mathrm{~cm}$ diameter side opening to allow the snake in and out, filled with a $2 \mathrm{~cm}$ layer of moist vermiculite. Laying dates spanned from the 17th of July to the 12th of August 2012 (N. maura) and from the $7^{\text {th }}$ of July to the $24^{\text {th }}$ of July 2012 (N. natrix). A total of 231 N. maura eggs $($ mean clutch size $=13.3 \pm 5.7)$ and $156 N$. natrix eggs $($ mean clutch size $=22.3 \pm$ 6.8) were obtained. For the purpose of the current experiment, we randomly selected a number of eggs from each $N$. maura clutch (50 eggs in total; $3.1 \pm 2.4$ eggs per clutch) and each $N$. natrix clutch ( 28 eggs in total; $\mathrm{N}=4.0 \pm 4.5$ per clutch). All females were returned to their exact site of capture within two weeks of egg-laying. From capture to release, females were offered small dead trouts once a week (frozen trouts were purchased from a local fish farm: Pisciculture des Chutes d'Aston, Les cabannes 09310).

Data collection. Eggs were collected within 12 hours of laying, placed on a $2 \mathrm{~cm}$ layer of wet vermiculite and transferred into an Aqualytic ${ }^{\circledR}$ incubation chamber set at a constant $28^{\circ} \mathrm{C}$. Eggs were individually marked with a letter (coding for litter of origin) and a number (egg number within each litter) for identification purposes. Eggs were measured in mass to the nearest $0.01 \mathrm{~g}$ using a digital scale within 12 hours of laying, and then every 7 days throughout the incubation period. Hatching occurred between the $19^{\text {th }}$ of August and the $6^{\text {th }}$ of September 2012 (N. natrix), and between the $21^{\text {st }}$ of August and the $25^{\text {th }}$ of September 2012 (N. maura). Hatchlings were measured within 12 hours of hatching in body mass $( \pm 0.01 \mathrm{~g})$, and snout-vent length to the nearest $0.1 \mathrm{~mm}$. Hatchlings were sexed by eversion of the hemipenes and marked by scaleclipping for identification. Siblings were housed together in plastic boxes $(15 \mathrm{~cm} \times$ $10 \mathrm{~cm} \times 5 \mathrm{~cm}$ ), with a water dish, shelter and paper towel as substrate. Snakes were remeasured in body mass, snout-vent length and total length at 3 weeks old, after which they were given their first meal (small dead minnows ranging from $0.5 \mathrm{~g}$ to $1 \mathrm{~g}$; supplied by Armorvif ${ }^{\circledR}$ ). After completion of the measurements, all young snakes were released at the maternal capture site.

Within each species, a body condition index ${ }^{23}$ (BCI) was calculated for each snake, using the residual values of the linear least-squares regression of Log (body mass) against Log (snout-vent length).

Capture, breeding, experimentation, release and ethics permits for N. maura and N. natrix were delivered by the Préfecture de l'Ariège (Arrété \#2012-11). All experiments were performed in accordance with the relevant guidelines and regulations.

Heart rate measurements. We estimated resting metabolic rate by measuring snakes' heart rates, owing to the accurate physiological relationship existing between heart rate and $\mathrm{VO}_{2}{ }^{24}$. We measured embryo heart rates as well as hatchling heart rates using the Buddy ${ }^{\circledR}$ digital egg monitor (MK2, Avitronics) under the protocol described for eggs $^{14}$ and neonate snakes ${ }^{13}$. The Buddy system works by shining an infrared beam onto the surface of the egg, detecting minute distortions caused by embryonic heart beats. Embryo heart rates were measured first at incubation day 10, and then every 10 days until hatching. Hatchling heart rates were measured at age $6.8 \pm 4.2$ days in a sub-sample of 22 juveniles snakes ( $15 \mathrm{~N}$. maura and $7 \mathrm{~N}$. natrix) and at age $=24.3 \pm$ 3.7 days $(\mathrm{N}=79)$. Snakes were placed inside individual testing bags (made of cotton material) fitted to their size, and into temperature controlled chambers for $30 \mathrm{~min}$ in order to reach testing temperature $\left(28^{\circ} \mathrm{C}\right)$ and ensure minimum stress levels at the time of testing ${ }^{13}$. Each bag was then placed onto the sensor pad of a Buddy ${ }^{\circledR}$ digital egg monitor (MK2, Avitronics) for heart rate reading (a stable reading was obtained after approximately 30 seconds)

Statistical analysis. All numerical data were Log-transformed prior to analysis. Data were checked for normality using Shapiro-Wilk W tests. Data analysis was performed using two factor repeated measures ANOVAs, post-hoc Tukey HSD and Linear regressions. Means \pm standard deviations are given unless otherwise stated.

1. Birchard, G. F. \& Reiber, C. L. Heart rate during development in the turtle embryo: Effect of temperature. J. Comp. Physiol. B 166, 461-466 (1996).

2. Birchard, G. F. An ontogenetic shift in the response of heart rates to temperature in the developing snapping turtle (Chelydra serpentina). J. Thermal Biol. 25, 287-291 (2000).
3. Nechaeva, M. V., Vladimirova, I. G. \& Alekseeva, T. A. Oxygen consumption as related to the development of the extraembryonic membranes and cardiovascular system in the European pond turtle (Emys orbicularis) embryogenesis. Comp. Biochem. Physiol. A 148, 599-610 (2007).

4. Du, W. G., Zhao, B. \& Shine, R. Embryos in the fast lane: high-temperature heart rates of turtles decline after hatching. PLoS ONE 5, e9557 (2010).

5. Angilletta, M. J., Lee, V. \& Silva, A. C. Energetics of lizard embryos are not canalized by thermal acclimation. Physiol. Biochem. Zool. 79, 573-580 (2006)

6. Radder, R. \& Shine, R. Thermally induced torpor in fullterm lizard embryos synchronizes hatching with ambient conditions. Biol. Letters 2, 415-416 (2006)

7. Pearson, J. T. \& Tazawa, H. Ontogeny of heart rate in embryonic and nestling crows (Corvus corone and Corvus macrorhynchos). J. Comp. Phys. B 169, 256-262 (1999).

8. Pough, E. H. et al. Herpetology ((Englewood Cliffs, Prentice-HallEnglewood Cliffs, Prentice-Hall, 1998).

9. Bobyn, M. L. \& Brooks, R. J. Incubation conditions as potential factors limiting the northern distribution of snapping turtles, Chelydra serpentina. Can. J. Zool. 72, 28-37 (1994).

10. Warner, D. A. \& Shine, R. Fitness of juvenile lizards depends on seasonal timing of hatching, not offspring body size. Oecologia 154, 65-73 (2007)

11. Pike, D. A., Pizzatto, L., Pike, B. A. \& Shine, R. Estimating survival rates of uncatchable animals: the myth of high juvenile mortality in reptiles. Ecology 89, 607-611 (2008)

12. Olsson, M. \& Shine, R. Growth to death in lizards. Evolution 56, 1867-1870 (2002).

13. Aubret, F., Tort, M. \& Blanvillain, G. A non-invasive method of measuring heart rates in small reptiles and amphibians. Herp. Review 44, 421-423 (2013).

14. Du, W. G., Radder, R. S., Sun, B. \& Shine, R. Determinants of incubation period: do reptilian embryos hatch after a fixed total number of heart beats? J. Exp. Biol. 212, 1302-1306 (2009)

15. Ar, A. \& Tazawa, H. Analysis of heart rate in developing bird embryos: effects of developmental mode and mass. Comp. Biochem. Physiol. A 124, 491-500 (1999).

16. Du, W. G., Ye, H., Zhao, B., Pizzatto, L., Ji, X. \& Shine, R. Patterns of interspecific variation in the heart rates of embryonic reptiles. PloS ONE 6, e29027 (2011).

17. O’Donoghue, M. \& Boutin, S. Does reproductive synchrony affect juvenile survival rates of northern mammals? Oikos 74, 115-121 (1995).

18. Vitt, L. J. Ecology and life history of the scansorial arboreal lizard Plica plica (Iguanidae) in Amazonian Brazil. Can. J. Zool. 69, 504-511 (1991).

19. Arnold, S. J. \& Wassersug, R. J. Differential predation on metamorphic anurans by garter snakes (Thamnophis): social behavior as a possible defense. Ecology 59 1014-1022 (1978).

20. Dehn, M. M. Vigilance for predators: detection and dilution effects. Behav. Ecol. Sociobiol. 26, 337-342 (1990).

21. Gasc, J. P. et al. Atlas of Amphibians and Reptiles in Europe (Muséum National d'Histoire Naturelle \& Service du Patrimoine Naturel, Paris, 1997).

22. Hailey, S., Davies, P. M. C. \& Pulford, E. Lifestyle and thermal ecology of natricine snakes. Brit. J. Herp. 6, 261-268 (1982).

23. Bonnet, X. \& Naulleau, G. Utilisation d'un indice de condition corporelle (BCI) pour l'étude de la reproduction chez les serpents. Comptes rendus de l'Académie des sciences. Série 3, Sciences de la vie 317, 34-41 (1994).

24. Butler, P. J., Green, J. A., Boyd, I. L. \& Speakman, J. R. Measuring metabolic rate in the field: the pros and cons of the doubly labelled water and heart rate methods. Funct. Ecol. 18, 168-183 (2004).

\section{Acknowledgments}

I wish to thank numerous field and laboratory helpers: Mélodie Tort, Tom Sarraude, Thomas Achkar, Alice Thiney and Gaëlle Blanvillain. I also wish to thank the CNRS, the Université Paul Sabatier for funding (Project EXUVIE), and the Agence Nationale pour la Recherche. This work is part of the ANR INDHET. There is no conflict of interest associated with this manuscript. This work is part of the "Laboratoire d'Excellence" (LABEX) entitled TULIP (ANR -10-LABX-41)

\section{Author contributions}

F.A. collected, analysed the data, and wrote the article.

\section{Additional information}

Competing financial interests: The authors declare no competing financial interests.

How to cite this article: Aubret, F. Heart rates increase after hatching in two species of natricine snakes. Sci. Rep. 3, 3384; DOI:10.1038/srep03384 (2013).

(i) $(-)$ This work is licensed under a Creative Commons Attributionvisit http://creativecommons.org/licenses/by-nc-nd/3.0 